\title{
Intoxicaciones masivas con plaguicidas en Colombia
}

\author{
Alvaro Javier Idrovo
}

\begin{abstract}
Resumen
Este artículo resume los hechos de las tres intoxicaciones masivas con plaguicidas ocurridas en Colombia hasta 1998. La primera ocurrió en Chiquinquirá (Boyacá) en 1967 e involucró a más de 500 personas, de los cuales 165 requirieron tratamiento hospitalario y 63 murieron. La segunda ocurrió en Puerto López (Meta) en 1970 y tuvo 190 intoxicados, de los cuales 157 requirieron tratamiento médico y 7 murieron. El tercer caso ocurrió en Pasto (Nariño) en 1977 y tuvo como consecuencia más de 300 intoxicados, con 120 hospitalizados y 15 muertos.

En los casos de Chiquinquirá y Pasto los plaguicidas que provocaron la intoxicación fueron metil-paratión y paratión respectivamente, mientras que en el caso de Puerto López solo se conoce que fue un plaguicida organofosforado. Los 3 casos de intoxicación masiva ocurrieron por consumo de alimentos contaminados.
\end{abstract}

Estos casos se analizaron y se compararon con las principales intoxicaciones ocurridas en el mundo. Se propone una discusión en torno a las consecuencias que tuvieron estos hechos en las políticas de salud pública nacionales, la capacidad de hacer frente a situaciones de este tipo en el país, la capacitación de personal para la prevención y manejo de estas situaciones y la educación de los profesionales de la salud en epidemiología, toxicología y otras disciplinas involucradas en el campo de la salud ambiental.

\section{Large-scale Pesticide Poisoning in Colombia}

\section{Summary}

This article reviews large-scale pesticide poisoning which has occurred in Colombia on three separate occasions up to 1998. The first one happened in Chiquinquirá (Boyacá) in 1967. More than five hundred people suffered from poisoning; one hundred and sixtyfive required treatment and sixty-three died. The second outbreak was in Puerto Lopez (Meta), in 1970, when one hundred and ninety people were intoxicated, one hundred and fifty seven required treatment and seven died. The third one occurred in Pasto (Nariño), in 1977, when more than three hundred people were intoxicated, one hundred and twenty of them required treatment and fifteen died.

The pesticides producing poisoning in Chiquinquirá and Pasto were methyl-parathion and parathion respectively. In Puerto López it is only known that the intoxication was caused by an organophosphorus pesticide. All three large-scale poisoning cases were due to contaminated food ingestion.

Laboratorio Salud Ambiental, Instituto Nacional de Salud; Departamento de Salud Pública y Tropical, Facultad de Medicina, Universidad Nacional de Colombia. 
These three cases are analysed and compared with world-wide poisonings. This article is the first step in the discussion of the impact which large-scale poisonings had on national public health policies, the actual capacity to handle similar situations, the need to improve management and prevention of similar situations and the ability to train professionals in the areas of environmental health, such as epidemiologists and toxicologists.

"Las epidemias son en la historia médica de los pueblos, los acontecimientos principales, los sucesos más notables. Se debe perpetuar su recuerdo a fin que las tristes lecciones de estas calamidades no sean olvidadas por las generaciones posteriores, para que no sean desconocidas por los médicos y por la población en general" Mauricio de Fleury.

La enfermedad y la muerte masiva de individuos cuyas causas han sido los productos químicos, hasta mediados del siglo XX, ocurrían principalmente entre algunos tipos de oficios o profesiones específicamente. Sin embargo, después de la segunda guerra mundial y debido al rápido crecimiento y expansión de la industria química, estos hechos empezaron a afectar a poblaciones no expuestas ocupacionalmente a estos agentes (1). Las intoxicaciones masivas por los plaguicidas constituyen un capítulo importante en esta historia, ya que han ocurrido graves hechos en muchas regiones del mundo, donde los plaguicidas han causado la muerte y la enfermedad de grandes poblaciones.

Un plaguicida es cualquier sustancia o mezcla de sustancias destinadas a prevenir, destruir o controlar cualquier plaga, incluyendo los vectores de enfermedades humanas o de animales, las especies no deseadas de plantas o animales que causan perjuicio o que interfieren de cualquier otra forma en la producción, elaboración, almacenamiento, transporte o comercialización de alimentos, productos agrícolas, madera y productos de madera o alimentos para animales, o que pueden administrarse a los animales para combatir insectos, arácnidos u otras plagas en o sobre sus cuerpos. El término incluye las sustancias destinadas a utilizarse como reguladoras del crecimiento de las plantas, defoliantes, desecantes, agentes para reducir la densidad de fruta o agentes para evitar la caída prematura de la fruta, y las sustancias aplicadas a los cultivos antes o después de la cosecha para proteger el producto contra la deterioración durante el almacenamiento y transporte (2). Entre los efectos adversos sobre la salud humana que han sido asociados con la exposición a plaguicidas se incluyen la intoxicación aguda, el cáncer, los trastornos en el sistema inmune, la neurotoxicidad, los trastornos en la reproducción, los trastornos de la médula ósea, las alteraciones citogenéticas, las dermatosis y la inducción enzimática (3).

Dentro de los plaguicidas, los insecticidas constituyen el grupo que tiene acción sobre los insectos; por esta razón, tienen una mayor probabilidad de afectar a los humanos. La historia de los insecticidas usualmente se ha dividido en dos eras: la era anterior al dicloro-difeniltricloroetano (DDT) y la era posterior al DDT. La primera era terminó en 1874 cuando Zeidler sintetizó el DDT, y se caracterizó por el uso de pocas sustancias usadas para el lavado de las plantas. Los principales agentes utilizados para el control de las plagas durante esta época fueron el sulfuro inorgánico, el arsénico, el arsenato de plomo, la criolita, y el ácido bórico, junto a sustancias vegetales como la piretrina, la nicotina, la rotenona, la rianodina y la veratridina. Posterior al desarrollo del DDT, desde la segunda década del siglo XX se empezó el estudio de la estructura y la síntesis de los insecticidas extraídos de las sustancias vegetales y, durante los años 30 , se inició la era de los insecticidas orgánicos sintéticos (4).

Los exitosos resultados del uso del DDT en el control de la epidemia de tifo transmitida por piojos ocurrida en Nápoles en 1944 incentivaron el uso de este compuesto alrededor del mundo en el control de la malaria y otras enfermedades transmitidas por vectores; además motivó la investigación de nuevos compuestos clorados con acción semejante entre los que se puede 
resaltar el lindano, que fue sintetizado con base en el hexaclorociclohexano (5). De esta manera, los clorados se convirtieron en los compuestos que dominaron en el segundo cuarto de siglo el control de las plagas de insectos (4).

En 1937, Schrader descubrió la acción insecticida y la utilidad como arma química de los organofosforados al inhibir la enzima colinesterasa. En la década de los 40, se inició la investigación de sustancias con acción insecticida con base en la fisostigmina logrando la síntesis del carbaril, el primer metil-carbamato con alta efectividad. Las lecciones más importantes de estos años ("tiempos de los insecticidas con acción en las colinesterasas") se pueden resumir en: i) la detoxificación más rápida en los mamíferos que en los insectos puede ser un factor de seguridad; ii) la toxicidad selectiva se puede lograr con el uso de proinsecticidas que tengan mayor actividad en los insectos que en los mamíferos; iii) existen importantes diferencias entre las especies en la acción de los organofosforados y los carbamatos insecticidas, así tengan un mismo blanco bioquímico; iv) algunos efectos tóxicos en los mamíferos se deben más a la exposición aguda que a la crónica o a los efectos acumulativos, v) en comparación con los clorados, los orgariofosforados y los carbamatos insecticidas son menos persistentes en el ambiente y más biodegradables, y vi) es posible preparar compuestos sistémicos que se movilicen internamente en las plantas después de una aplicación única y protejan la punta de crecimiento por varias semanas (4).

Junto al desarrollo de los plaguicidas fue creciendo la crítica a su uso. Es así como hasta finales de la década de los 50 , los plaguicidas fueron considerados como de poco o ningún peligro para la salud humana y el ambiente; posteriormente, la publicación del libro La primavera silenciosa ("Silent Spring") de Rachel Carson en 1962 (6), cambió de manera radical la forma de pensar del público en general frente a los plaguicidas. Aunque muchos de los argumentos que se exponen en contra de los plaguicidas en esta obra hacen referencia al DDT específicamente, el impacto fue sentido por la industria agroquímica en general, ocasionando la estigmatización de la palabra "plaguicida" como sinónimo de muerte y daño de la naturaleza y el hombre (4), sin que en la práctica se haya podido evidenciar una clara asociación entre la exposición a estas sustancias y muchos efectos adversos en la salud humana. Un hecho que vale la pena resaltar es que a mediados de la década de los 50 Luis María Murillo, pionero de la entomología colombiana, empezó a discutir acerca de los potenciales efectos nocivos de los plaguicidas sobre los ecosistemas y la salud del hombre (7).

Posteriormente, se desarrollaron otros tipos de insecticidas como los piretroides y las formamidinas, junto a otros plaguicidas con acción herbicida, fungicida o molusquicida como los ditiocarbamatos, las triazinas, las ureas sustituidas, los compuestos organotin, los carbamatos herbicidas y fungicidas, los fenoxiacetatos, los compuestos amonio cuaternarios y los derivados de la morfolina. La gran mayoría de estos presenta una toxicidad aguda muy inferior a la de los clorados y los organofosforados.

En Colombia poco se conocen o recuerdan los casos en que la contaminación por plaguicidas ha ocasionado enfermedad, incapacidad o muerte de grupos humanos. Por tal razón, el presente trabajo pretende recopilar brevemente la historia de las intoxicaciones masivas por estos compuestos hasta 1998. Para tal fin, se consultaron los informes técnicos, las publicaciones científicas nacionales e internacionales y los periódicos de circulación nacional hasta 1998 donde se informara de intoxicaciones masivas con plaguicidas. Los criterios para la selección de los casos fueron que el agente, conocido o probable, fuera un plaguicida e involucrara por lo menos 100 personas. El resultado de esta búsqueda se encuentra en el cuadro 1.

\section{El caso de Chiquinquirá (1967) (8-11)}

Si bien el mundo no se había repuesto de los trágicos episodios de intoxicación masiva por consumo de pan hecho con harina contaminada con plaguicidas durante su transporte, ocurridos 
Cuadro 1. Intoxicaciones masivas con plaguicidas en Colombia hasta 1998.

\begin{tabular}{|c|c|c|c|c|c|}
\hline \multirow[b]{2}{*}{ Lugar } & \multirow[b]{2}{*}{ Año } & \multirow[b]{2}{*}{ Agente } & \multicolumn{3}{|c|}{ Intoxicados } \\
\hline & & & Hospitalizados & Muertos & Total* \\
\hline Chiquinquirá, Boyacá & 1967 & Metil-paratión & 165 & 63 & $>500$ \\
\hline Puerto López, Meta & 1970 & Organofosforado** & 157 & 7 & 190 \\
\hline Pasto, Nariño & 1977 & Paratión & 120 & 15 & $>300$ \\
\hline
\end{tabular}

* Cálculo aproximado.

** Probablemente, paratión o metil-paratión.

en 1967 en Doha (Qatar), Hofuf (Arabia Saudita) y México, en Chiquinquirá ocurrió un caso en el cual murieron aún más individuos que en los anteriores incidentes (12). Todo empezó el 24 de noviembre de 1967 cuando un camión salió de Bogotá con destino Chiquinquirá llevando 30 bultos de harina, 4 bultos de salvado, 29 cajas de folidol metil-paration y 2 cajas de antracol (propineb). Según declaraciones del conductor del camión hechas después de ocurridos los hechos, debido al mal estado de la carretera un frasco de $1000 \mathrm{cc}$ con Folidol al 50\% se rompió e impregnó algunos bultos de harina. Todo el cargamento de harina y salvado fue entregado en la panadería Nutibara, de Chiquinquirá, donde se procedió a preparar pan para venderlo el día siguiente.

El sábado 25 de noviembre este pan fue vendido a un almacén, una tienda y varias personas particulares. Hacia las 8 de la mañana comenzaron a llegar al Hospital San Salvador las primeras personas intoxicadas; su número fue en aumento hasta llegar a 165 personas, de las cuales 63 fallecieron, siendo la mayoría de los afectados menores de 15 años $(60,9 \%)$. El cuadro clínico fue muy variado; dolor abdominal, temblor, vértigo y astenia se presentó en más de $25 \%$ de los individuos; sintomas de intoxicación aguda como lagrimeo, visión borrosa y somnolencia en $2 \%$; midriasis y edema pulmonar agudo en $5,9 \%$; sialorrea en $7,8 \%$; diarrea y cianosis en $9,8 \%$; y disnea, sudoración, parestesias, disestesias y miosis en $11,8 \%$ de los casos. Antes del mediodía ya habían fallecido 62 de los individuos intoxicados, cuadro 2. La situación en el Hospital fue caótica; no había suficientes médicos, enfermeras, camas ni elementos para la atención de los pacientes que iban llegando, por lo cual fue necesario ubicar varios individuos en una misma cama y utilizar los corredores para albergar al resto. El tratamiento inicial fue el lavado gástrico, seguido por el consumo de clara de huevo y leche, los cuales eran considerados como sustancias neutralizantes de los residuos tóxicos (13).

Las primeras sospechas indicaban que el agente causante de la tragedia era el arsénico presente en el agua del acueducto municipal, lo que conllevó a prohibir el consumo de agua y la necesidad de buscar el tratamiento específico para este tipo de intoxicación. Como respuesta ante esta situación, el gobierno nacional envió una comisión investigadora, junto a 35 médicos y 83 enfermeras. La comisión estuvo conformada por personal del Ministerio de Salud, el Hospital de Tunja y el Instituto de Medicina Legal. Dicha comisión partió de Bogotá en helicóptero llevando 400 ampollas de BAL (dimercaprol) y llegó a Chiquinquirá hacia las 2 de la tarde.

Inmediatamente llegaron los comisionados, se revisaron las historias clínicas de los intoxicados y se empezó el análisis del contenido gástrico de un paciente hospitalizado, del pan ingerido, del contenido gástrico de un individuo fallecido, una muestra de harina proveniente de un bulto contaminado, un pedazo de talego de la panadería Nutibara y una muestra de agua de la red del acueducto municipal, en búsqueda de plaguicidas organofosforados, principales sospechosos desde el primer momento debido a la historia clínica y el olor característico emanado de las muestras. Los resultados de los análisis de estas muestras solo se conocieron 5 días después. 
Cuadro 2. Distribución de los fallecidos y hospitalizados en la intoxicación masiva de Chiquinquirá (1967) según edad y sexo $(10,11)$.

\begin{tabular}{|c|c|c|c|c|c|c|c|c|c|}
\hline \multirow{2}{*}{$\begin{array}{l}\text { Edad } \\
\text { (años) }\end{array}$} & \multicolumn{3}{|c|}{ Hospitalizados } & \multicolumn{3}{|c|}{ Fallecidos } & \multicolumn{2}{|c|}{ Total } & \multirow[b]{2}{*}{$\%$} \\
\hline & Hombres & Mujeres & $\%$ & Hombres & Mujeres & $\%$ & Hombres & Mujeres & \\
\hline$<5$ & 9 & 9 & 10,9 & 7 & 7 & 22,2 & 16 & 16 & 14,0 \\
\hline $5-9$ & 14 & 18 & 19,4 & 17 & 11 & 44,5 & 31 & 29 & 26,3 \\
\hline $10-14$ & 14 & 20 & 20,6 & 8 & 5 & 20,6 & 22 & 25 & 20,6 \\
\hline $15-19$ & 12 & 13 & 15,2 & 2 & 2 & 6,3 & 14 & 15 & 12,7 \\
\hline $20-24$ & 6 & 6 & 7,3 & - & - & - & 6 & 6 & 5,3 \\
\hline $25-29$ & 3 & 9 & 7,3 & 1 & 1 & 3,2 & 4 & 10 & 6,1 \\
\hline $30-34$ & 3 & 5 & 4,8 & - & - & - & 3 & 5 & 3,5 \\
\hline $35-39$ & 1 & 6 & 4,3 & - & 1 & 1,6 & 1 & 7 & 3,5 \\
\hline $40-44$ & - & 4 & 2,4 & - & - & - & - & 4 & 1,8 \\
\hline $45-49$ & 2 & 2 & 2,4 & - & - & - & 2 & 2 & 1,8 \\
\hline $50-54$ & 4 & 1 & 3,0 & - & - & - & 4 & 1 & 2,2 \\
\hline $55-59$ & 1 & - & 0,6 & - & - & - & 1 & - & 0,4 \\
\hline $60-64$ & - & 1 & 0,6 & 1 & - & 1,6 & 1 & 1 & 0,9 \\
\hline $65<$ & - & 2 & 1,2 & - & - & - & - & 2 & 0,9 \\
\hline Total & 69 & 96 & 100,0 & 36 & 27 & 100,0 & 105 & 123 & 100,0 \\
\hline
\end{tabular}

Pese a que no se habían realizado los análisis químicos que plenamente identificaran al agente causal de la intoxicación, hacia las 2:30 de la tarde se inició el tratamiento con atropina de manera sistemática, permitiendo la pronta recuperación de la mayoría de los individuos que aún permanecían intoxicados; posteriormente, se inició el uso de pralidoxima (2-PAM), cuando pudo ser llevada desde ciudades como Medellín. Además de estos medicamentos presentes en el país, llegó toxogonina proveniente de Panamá y Caracas, que al parecer no fue utilizada en el tratamiento de las víctimas.

Para evitar otros casos de intoxicación, se decidió quemar toda la harina y pan que se encontrara en la ciudad. Sin embargo, la población en general siguió sintiéndose amenazada y prefirió no consumir alimentos en todo el día.

Como consecuencia de la intoxicación, también se vivió una fuerte controversia entre los investigadores y médicos que atendieron la emergencia y un químico farmacéutico, quien sostenía que el BAL enviado a Chiquinquirá fue la causa de la muerte de muchos de los intoxicados. Sin entrar en detalles, el desenlace de esta disputa es que al parecer nunca se uso el BAL debido a que su llegada fue posterior a la muerte de 62 de los 63 intoxicados que fallecieron.

\section{El caso de Puerto López $(1970)(14,15)$}

Este caso sólo pudo conocerse a través de los informes de los periódicos; por tal razón sus datos no tienen un fuerte sustento técnico y están sujetos a grandes limitaciones. Hacia las 8:30 de la mañana del 2 de enero de 1970, un vecino de la vereda La Balsa del municipio de Puerto López (Meta), se presentó en la alcaldía municipal para informar la muerte de 2 adultos y la intoxicación de los 2 hijos de estos. Ante esta situación, el alcalde decidió enviar al inspector de policía para que coordinara el levantamiento de los cadáveres y el transporte de los enfermos al hospital del municipio. Momentos después llegó información de numerosos casos de intoxicados, lo que hizo necesario comunicarse con las autoridades sanitarias departamentales en Villavicencio para solicitar su colaboración. Con esta misión partieron de Villavicencio con rumbo a Puerto López 5 médicos y 3 bacteriólogas a cargo del director del hospital de Villavicencio, para manejar los intoxicados.

Durante todo el día, llegaron a la cabecera municipal 157 intoxicados procedentes de las veredas La Balsa, San Luis, La Ponderosa, 
Yiacuanas y bocas de río Negro para ser atendidos en la iglesia y en los centros educativos que fueron acondicionados para tal fin. Todo el municipio fue presa del pánico y por miedo a consumir cualquier tipo de alimento contaminado solo se consumió durante ese día y los siguientes gaseosa y cerveza.

El diagnóstico de la intoxicación por plaguicida inhibidor de la colinesterasa se realizó, y exigió la colaboración de todas las instituciones gubernamentales de la región y la Cruz Roja para la movilización de fármacos. Para el 4 de enero, fueron dados de alta todos los que habían sido hospitalizados dejando como saldo 7 individuos muertos y 190 intoxicados. Las investigaciones indicaron como la causa de este episodio a la contaminación del río Negro con plaguicidas organofosforados y la posterior muerte masiva de peces, el 31 de diciembre, y su consumo por los habitantes de la cuenca los días 1 y 2 de enero. El plaguicida implicado no fue claramente identificado, pero se sospechó que hubiera sido paration o metil-paratión, ya que estos eran los comúnmente utilizados en los cultivos de arroz de la región. Cabe resaltar que desde el año anterior se había denunciado que la fumigación aérea de estos cultivos se hacía con poca precaución y que los fumigadores lavaban sus equipos en los ríos.

\section{El caso de Pasto $(1977)(16,17)$}

Pese a que en publicaciones nacionales se refiere este caso $(18,19)$, en ningún lugar se encuentra documentado de manera técnica este trágico episodio. La reconstrucción de los hechos permite decir que se inició cuando un cargamento de harina contaminada con paratión proveniente del Ecuador fue introducido al país de contrabando y distribuido en las panaderías de la ciudad.

El 12 de septiembre de 1977 en una panadería del barrio Bernal de Pasto se fabricó pan, en la ciudad con esta harina. Hacia las 5:30 de la tarde empezaron a presentarse los primeros casos de intoxicación, cuyos síntomas principales fueron los del aparato digestivo, entre los que sobresalió el dolor abdominal. La situación desencadenó una ola de pánico por toda la ciudad; en el Hos- pital Departamental, el Hospital Infantil Los Angeles y la Clínica San Pedro. Rápidamente se hizo el diagnóstico de intoxicación por plaguicida inhibidor de las colinesterasas con lo cual prontamente se inició el tratamiento con sulfato de atropina. La zona donde hubo más casos de intoxicados, comprendió los barrios Bernal, Mercedario, Lorenzo, Santa Bárbara, La Rosa, Venecia, Corazón de Jesús y Centro.

La crítica situación hizo que todos los médicos de Pasto colaboraran en el tratamiento de los enfermos, que la policía implementara una operación de salvamento, que ayudas de fármacos fueran enviadas desde Cali, Popayán y Bogotá para controlar la situación, y que el alcalde, por recomendaciones del director del Hospital San Pedro, prohibiera el consumo de pan en toda la ciudad.

Los análisis químicos, realizados por el departamento de química de la Universidad de Nariño y por el laboratorio de la zona minera de Pasto, permitieron identificar al paratión como el agente causal de las intoxicaciones. Debido a estos hechos, el ejército colombiano reforzó las medidas de control en el sur del departamento de Nariño como medida preventiva para evitar el paso de nuevos cargamentos de alimentos contaminados con plaguicidas provenientes del Ecuador.

\section{Discusión}

El presente trabajo resume los 3 episodios de intoxicación masiva por plaguicidas ocurridos en Colombia hasta 1998; sin embargo, los datos aquí registrados no reflejan la real magnitud de las intoxicaciones agudas con este tipo de agentes en el país. Falta añadir a estos casos los que han ocurrido en ambientes laborales, homicidios, suicidios y las intoxicaciones accidentales de grupos pequeños de individuos $(20,21)$. Al comparar los casos ocurridos en Colombia con los registrados en otros países (Cuadro 3), se puede observar que el caso de Chiquinquirá se encuentra entre los 5 que más muertos han provocado; los episodios de Chiquinquirá y Pasto se encuentran entre los 10 con mayor número de intoxicados y que los 3 casos colombianos se encuentran entre los 15 
Cuadro 3. Principales casos de intoxicación masiva aguda con plaguicidas en países diferentes a Colombia (22-38).

\begin{tabular}{|c|c|c|c|c|c|}
\hline Lugar & Año & Agente & Grupo & Intoxicados & Muertos \\
\hline Estados Unidos & 1943 & Fluroruro de Sodio & & 260 & 47 \\
\hline Glamorgan, Reino Unido & 1954 & Endrin & OC & $>100$ & sd \\
\hline Wales, Reino Unido & 1956 & Endrin & OC & 59 & 0 \\
\hline Kerala, India & 1958 & Paratión & OF & 828 & 106 \\
\hline El Cairo, Egipto & 1958 & Paratión & OF & 200 & 8 \\
\hline Singapur & 1960 & Paratión & OF & 38 & 9 \\
\hline Iraq & 1960 & Etil-mercurio & & $1000^{*}$ & sd \\
\hline Guatemala & $1963-5$ & Metil-mercurio & & 45 & 20 \\
\hline Doha, Qatar & 1967 & Endrin & $\mathrm{OC}$ & $490(h)$ & 7 \\
\hline Doha, Qatar & 1967 & Endrin & OC & $188(h)$ & 17 \\
\hline Hofuf, Arabia Saudita & 1967 & Endrin & OC & $183(h)$ & 2 \\
\hline México & 1967 & Paratión & OF & $>100$ & 15 \\
\hline Iraq & $1971-2$ & Metil-mercurio & & $6530(\mathrm{~h})$ & $459(h)$ \\
\hline Janes River, Estados Unidos & $1973-5$ & Clordecone & $O C$ & 148 & \\
\hline Jamaica & 1975 & Paratión & OF & 62 & 17 \\
\hline Pakistán & 1976 & Malatión & OF & 2800 & 5 \\
\hline Bhopal, India & 1984 & Isocianato** & & $8000^{*}$ & $1048^{*}$ \\
\hline Estados Unidos y Canadá & 1985 & Aldicarb & $\mathrm{Cl}$ & 1376 & 0 \\
\hline Singapur & 1988 & $\begin{array}{l}\text { Metamidofos y } \\
\text { Propenofos }\end{array}$ & OF & 105 & 0 \\
\hline
\end{tabular}

OC: Organoclorado, OF: Organofosforado; Cl: Carbamato insecticida; (h): datos de hospitales únicamente; sd: sin dato.

* Cálculo aproximado en versiones oficiales.

** Si bien el isocianato no es un plaguicida, en este caso estaba siendo utilizado en la síntesis de carbaril (Cl).

primeros en términos de individuos intoxicados en general y que requirieron tratamiento. hospitalario.

En Colombia, los agentes involucrados en las intoxicaciones masivas por plaguicidas siempre han sido los compuestos organofosforados, mientras que a nivel mundial son en orden descendente los organofosforados, los carbamatos, los organoclorados y los compuestos mercuriales orgánicos (39). El paratión y el metil-paratión fueron claramente identificados en los episodios de Pasto y Chiquinquirá, respectivamente, mientras que en el caso de Puerto López solo se sospecha que fue alguno de estos agentes; estos plaguicidas organofosforados tienen acción sobre los insectos, siendo considerados como extremadamente peligrosos, clase la de la OMS, teniendo unas dosis letal (DL50) 50 orales de $13 \mathrm{mg} / \mathrm{kg}$ para el paratión y de $14 \mathrm{mg} / \mathrm{kg}$ para el metilparatión $(40,41)$.

Según Levine y Doull, entre 1951 y 1990 se habían registrado 148 brotes epidémicos por causa de los plaguicidas en todo el mundo, ocasionando 24.731 intoxicados y 1.065 muertes. Estos datos se consideran subestimados debido a que no se han reportado todos los casos y, como ejemplo, basta citar el caso ocurrido en Puerto López. Pese a esta limitación, se ha podido establecer que la vía más común de exposición ha sido a través de los alimentos y que los países con mayor número de episodios son Estados Unidos y Tailandia (39).

Como característica común en el manejo de los tres episodios de intoxicación masiva con plaguicidas en Colombia, se encuentra el predominio del enfoque clínico o de tratamiento individualizado de los sujetos intoxicados. No se 
pudo apreciar la interacción de múltiples expertos (epidemiólogos, bioestadísticos, médicos clínicos, patólogos, expertos en métodos analíticos, toxicólogos ambientales, psicólogos y antropólogos), los cuales deberían integrar el equipo que se dedique a la identificación de la fuente de contaminación, la medición y estimación de la exposición de los individuos y la población en general, el impacto del evento sobre la salud humana (aspectos biológicos y psicosociales), y las medidas clínicas, administrativas, políticas y técnicas de respuesta frente a la intoxicación masiva (1).

Antes del caso de Chiquinquirá se habían descrito varios casos similares en otros países en los que la contaminación del alimento ocurrió durante el transporte. El primero ocurrió en Glamorgan (Reino Unido), en el cual durante el transporte por tren se contaminó con endrin la harina que se llevaba en el mismo vagón. Con esta harina se hicieron pasteles y pan que fueron consumidos por habitantes del valle Swansea, ocasionando más de 100 intoxicados. Los otros casos, al igual que el de Chiquinquirá, ocurrieron en 1967 en Doha (Qatar) y Hofuf (Arabia Saudita). Estos casos íntimamente relacionados ocurrieron por el almacenamiento en un mismo compartimento de alimentos y plaguicidas (26).

En este caso, debido a que fue el primero en el país, se pueden hacer algunas consideraciones acerca del tratamiento clínico recibido por los intoxicados. Si bien el desarrollo de la toxicología clínica en esta época era incipiente, ya existían documentos que muestran el interés en el tema y de manera muy especial en el paratión (42). Llama la atención que el tratamiento inicial fuera el lavado gástrico seguido por la ingestión de clara de huevo y leche, mientras que el diagnóstico inmediato de la comisión enviada fue el de intoxicación por plaguicida organofosforado, lo que permitió iniciar el tratamiento con atropina. Lo que muestra este hecho es la falta de preparación que los profesionales de salud de aquella época tenían en toxicología.

En el incidente de Puerto López no se conoció con certeza la forma en la cual se contaminó el río Negro. Sin embargo, el conocimiento que la zona era aprovechada para el cultivo de arroz y que en este tipo de cultivo era frecuente el uso de paratión y metil-paratión, permite afirmar con un alto grado de certeza que ambos o alguno de estos plaguicidas fue la causa de la intoxicación. La contaminación ambiental de lagos, ríos y bahías con plaguicidas organofosforados y la consecuente muerte de organismos acuáticos ha sido descrita en varias regiones del mundo (41). La dosis necesaria para la muerte de los peces depende de la especie y tamaño del animal. Como en los ríos de la zona del Meta y en general de la Orinoquia son frecuentes los peces payaras, cachamas, rayados, sapuaras, mapurites, nicuros y amarillos, la dosis solo pudo haberse logrado por el vertimiento del plaguicida directamente en el agua, ya que en la fumigación aérea el viento puede transportar el compuesto hasta $2 \mathrm{~km}$ del lugar de fumigación. Además, si el reporte de la mortandad de peces ocurrió el 31 de diciembre, es probable que el vertimiento haya ocurrido el mismo día o el anterior debido a que los plaguicidas organofosforados no son estables en el medio acuoso y solo son tóxicos durante el tiempo en el cual no se ha completado su hidrólisis (43).

El caso de Pasto aunque es similar al de Chiquinquirá tiene como característica particular el hecho que los plaguicidas traspasaron las fronteras del país. De esta manera, se evidencia claramente que las sustancias peligrosas para la salud humana no respetan los límites administrativos y que pueden provocar nefastas consecuencias más allá del país de origen. Por tal razón, es imperioso tener unas políticas claras respecto a la importación y exportación de dichas sustancias para evitar el paso de materiales no permitidos por su peligrosidad.

Los tres casos brevemente reseñados en este documento y los múltiples casos de intoxicación con plaguicidas en todo el mundo tuvieron que ocurrir para que por fin los organismos internacionales y los países empezaran a reglamentar su uso. Es así como, en 1985, la FAO expide el Código Internacional de Conducta para el uso de plaguicidas, documento que sirvió de fundamento del decreto 1843 de 1991 que regula dicha materia en el país, que ya había 
sido mencionada como importante en la Ley 9 de 1979 (44-45).

Para finalizar vale la pena reflexionar acerca de varios puntos:i) ¿Colombia tiene la capacidad de hacer frente a situaciones de intoxicaciones masivas con sustancias peligrosas?, ii) ¿Existe suficiente personal capacitado en la prevención y manejo de intoxicaciones masivas con plaguicidas?, iii) ¿Qué impacto han tenido estos casos en las políticas de salud pública nacionales de manejo de sustancias peligrosas?, iv) ¿Los profesionales de la salud reciben suficiente instrucción en toxicología?, y v) ¿Se están reportando adecuadamente todos los casos de intoxicación por agentes exógenos en Colombia?

La infraestructura nacional en los campos de toxicología y epidemiología ambiental aún sigue siendo incipiente, las políticas en salud pública en materia de sustancias peligrosas ha volcado casi todos los esfuerzos a la prevención de los efectos adversos de los plaguicidas y olvidado a toda una amplia gama de otros agentes químicos muchas veces más peligrosos. Sin duda alguna, el país aún tiene mucho que aprender de estas catástrofes para poder llegar a prevenir la ocurrencia de eventos futuros similares y lograr así una mejor salud de los colombianos, máxime cuando agentes químicos como los aquí presentados tienen el potencial de causar la muerte, enfermedad o incapacidad de miles de personas en un lapso muy corto de tiempo.

\section{Agradecimientos}

A la comunicadora social-periodista Alejandra Amaya por su apoyo en la búsqueda del material publicado en los diarios El Espectador y El Tiempo; a los compañeros del Laboratorio Salud Ambiental del Instituto Nacional de Salud y al doctor Mauricio Restrepo Trujillo por sus valiosos aportes al documento final.

\section{Referencias}

1. Bertazzi PA. Industrial disasters and epidemiology: a review of recent experiences. Scand JWork Environ Health 1989; 15: 85-100.

2. FAO. Código Internacional de Conducta para el uso de Plaguicidas. 28 de Noviembre de 1985.
3. AI Saleh IA. Pesticides: a review article. J Environ Pathol Toxicol Oncol 1994; 13: 151-61.

4. Casida JE, Quistad GB. Golden age of insecticide research: past, present, or future? Annu Rev Entomol 1998; 43: 1-16.

5. Packard RM. 'No other choice': global malaria eradication and the politics of international health in the post-war era. Parassitologia 1998; 40: 217-29.

6. Carson R. Silent Spring. Boston: Houghton Mifflin, 1962: 1-368.

7. Murillo LM. 30 años de sanidad vegetal - Historia de una vida. Rev Acad Colomb Cienc Exact Fis Quim Nat 1957; 10: V-XXII.

8. Garcia G. Chiquinquirá desayunó ayer con pan envenenado. El Espectador 1967 Noviembre 26; Pag. 9 A

9. Martínez M. 74 los muertos en Chiquinquirá. El Tiempo 1967 Noviembre 26:Pag.6

10. Galán R. Investigación epidemiológica de la intoxicación alimenticia presentada en el municipio de Chiquinquirá Trib Médica 1969; 34: 353-65.

11. Galán R. Investigación epidemiológica de la intoxicación alimenticia presentada en el municipio de Chiquinquirá [Informe Provisional]. Bogotá: Ministerio de Salud Pública, 1967.

12. Insecticides and gulf ports. Lancet 1968 (May 11): 1022.

13. González H, Rueda-Williamson R. Intoxicaciones exógenas. Rev Fac Med 1958; 26(10-11): 575-83.

14. Franco M.A. Cinco muertos en el Llano con aguas contaminadas. El Espectador 1970 Enero 3;pag. 1A.

15. Mosquera J. Dos muertos más por intoxicación en Meta. ElTiempo 1970 Enero 3;pag. 2.

16. Guerrero G. Quince muertos en Pasto por intoxicación masiva con alimentos. El Espectador 1977 Septiembre 13;Pag.1A.

17. Parra E. Pan con paratión causa 15 muertos, 115 hospitalizados. ElTiempo 1977 Septiembre 13:Pag.1A.

18. Restrepo M, Guerrero E. Los plaguicidas organofosforados. Revisión de sus aspectos médicos. Acta Med Colomb 1979; 4: 23-47.

19. Varona ME, Morales L, Ortiz J, Sánchez JF, Cárdenas O, De la Hoz F. Panorama epidemiológico de la exposición a plaguicidas inhibidores de colinesterasa en 17 departamentos del país. Biomédica 1998; 18: 22-9.

20. Henao S, Finkelman J, Albert L, Koning HW. Plaguicidas y salud en las Américas. México: Centro panamericano de Ecología Humana y salud, 1993.

21. García JE. Intoxicaciones agudas con plaguicidas: costos humanos y económicos. Rev Panam Salud Pública 1998; 4: 383-7.

22. Baker EL, Warren MW, Zack M, et al. Epidemic malathion poisoning in Pakistan malaria workers. Lancet 1978; Jan 7: 31-4. 
23. Bakir F, Damluji SF, Amin-Zaki L, et al. Methylmercury poisoning in Iraq. Science 1973; 181:230-41.

24. Coble Y, Hildebrandt P, Davis J, Raasch F, Curley A. Acute endrin poisoning. JAMA 1967; 202: 153-7.

25. Davidson W, Ellington AC, Manley D, et al. Foodborne pesticides poisoning - Jamaica. MMWR 1977; 26:317.

26. Davies GM, Lewis I. Outbreak of food-poisoning from bread made of chemically contaminated flour. BMJ 1956: 393-8.

27. Fernícola N. Aspecto toxicológico de la contaminación ambiental causada por accidentes. Bol Of Sanit Panam 1983; 95: 352-60.

28. Goes EA, Savage EP, Gibbons G, Aaronson M, Ford SA, Wheeler W. Suspected foodborne carbamate pesticide intoxications associated with ingestion of hydroponic cucumbers. Am J Epidemiol 1980; 111: 254-60.

29. Goh KT, Yew FS, Ong KH, Tan IK. Acute organophosphorus food poisoning caused by contaminated green leafy vegetables. Arch Environ Health 1990; 45: 180-4.

30. Goldman LR, Beller M, Jackson RJ. Aldicarb food poisonings in California, 1985-1988: toxicity estimates for humans. Arch Environ Health 1990; 45: 141-7.

31. Goldman LR, Smith DF, Neutra RR, et al. Pesticide food poisoning from contaminated watermelons in California, 1985. Arch Environ Health 1990; 45: 229-36.

32. Green MA, Heumann MA, Wehr HM, et al. An outbreak of watermelon-borne pesticide toxicity. Am J Public Health 1987; $77: 1431-4$.

33. Greenberg MI, Phillips SD. A brief history of occupational, industrial, and environmental toxicology. In: Greenberg Ml (editor). Occupational, industrial, and environmental toxicology. St. Louis: Mosby, 1997: 1-5.
34. Guthrie FE. Pesticides and humans. In: Guthrie FE, Perry JJ (editors). Introduction to environmental toxicology. New York: Elsevier, 1980: 299-312.

35. Hill RH, Alley CC, Ashley DL, et al. Laboratory investigation of a poisoning epidemic in Sierra Leone. J Anal Toxicol 1990; 14:213-6.

36. The Lancet. Calamity at Bhopal. Lancet 1984; 11(8416): 1378-9.

37. McConnell R, Hruska AJ. An epidemic of pesticide poisoning in Nicaragua: Implications for prevention in developing countries. Am J Public Health 1993; 83: 1559-62.

38. Weeks DE. Endrin food poisoning. Bull WHO 1967; 37 : 499-512.

39. Levine RS, Doull J. Global estimates for acute pesticide morbidity and mortality. Rev Environ Contam Toxicol 1992; 129: 29-50.

40. International Programme of Chemical Safety. The WHO recommended classification of pesticides by hazard and guidelines to classification 1996-1997. Geneva: UNEPILO-WHO, 1997.

41. Jeyaratnam J, Maroni M. Organophosphorous compounds. Toxicology 1994; 91: 15-27.

42. Hamblin DO, Goltz HH. Parathion. Envenenamiento Un breve resumen y prueba de colinesterasa y su aplicación en el campo. Bogotá: Ministerio de Salud Pública, 1956: 1-23.

43. World Health Organization. Environmental health criteria 63. Organophosphorus insecticides. Geneva: WHO, 1986.

44. Presidencia de la República de Colombia. Decreto 1843 (Julio 22 de 1991).

45. Congreso de Colombia. Ley 9, título III (Enero 24 de 1979). 